\title{
The closed knight tour problem in higher dimensions
}

\author{
Joshua Erde \\ Department of Pure Mathematics and Mathematical Statistics \\ University of Cambridge \\ Cambridge, CB3 0WB, United Kingdom \\ jpe28@cam.ac.uk \\ Bruno Golénia \\ Sylvain Golénia \\ Department of Computer Science \\ University of Bristol \\ Bristol BS8 1UB, United Kingdom \\ Institut de Mathématiques de Bordeaux \\ Université Bordeaux \\ goleniab@compsci.bristol.ac.uk \\ 33405 Talence cedex, France \\ sylvain.golenia@u-bordeaux1.fr
}

Submitted: Mar 20, 2012; Accepted: Oct 9, 2012; Published: Oct 25, 2012

Mathematics Subject Classifications: 05C45,00A08

\begin{abstract}
The problem of existence of closed knight tours for rectangular chessboards was solved by Schwenk in 1991. Last year, in 2011, DeMaio and Mathew provide an extension of this result for 3-dimensional rectangular boards. In this article, we give the solution for $n$-dimensional rectangular boards, for $n \geqslant 4$.
\end{abstract}

Keywords: Chessboard; Hamiltonian cycle

\section{Introduction}

On a chessboard, a knight moves by two squares in one direction and by one square in the other one (like an L). A classical challenge is the so-called knight tour. The knight is placed on the empty board and, moving according to the rules of chess, must visit each square exactly once. A knight tour is called a closed tour if the knight ends on a square attacking the square from which it began. If the latter is not satisfied and the knight has visited each square exactly once, we call it an open tour.

Some early solutions were given by Euler, see [4] and also by De Moivre (we refer to Mark R. Keen for historical remarks, see [7]). The problem was recently considered for various types of chessboards: as a cylinder [15], a torus [16], a sphere [1], the exterior of 


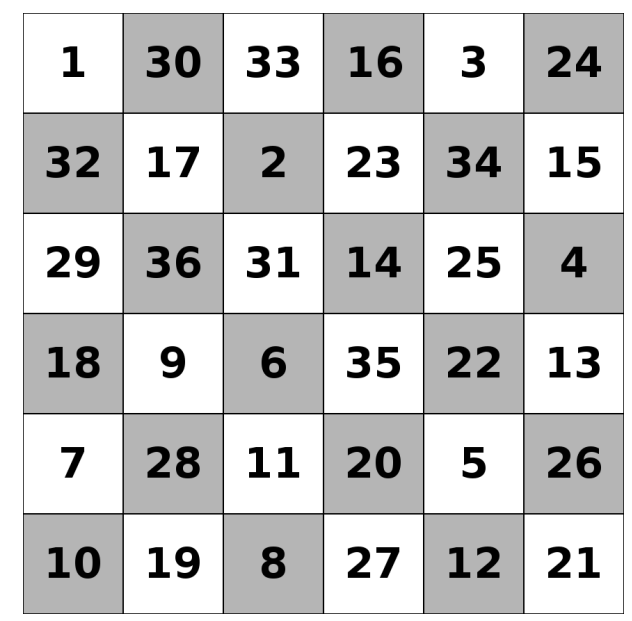

Figure 1: A $6 \times 6$ closed tour

the cube [11], the interior of the cube [2],.. It represents also an active field of research in computer science, e.g., [10] (see references therein). In this paper, we shall focus on rectangular boards.

In 1991, Schwenk considered the question of the closed knight tour problem in a 2dimensional rectangular chessboard. He provided a necessary and sufficient condition on the size of the board in order to have a closed knight tour. He obtained:

Theorem 1 (Schwenk). Let $1 \leqslant n \leqslant m$. The $n \times m$ chessboard has a closed knight tour if and only if the following conditions hold:

1. $n$ or $m$ is even,

2. $n \notin\{1,2,4\}$,

3. $(n, m) \notin\{(3,4),(3,6),(3,8)\}$.

We refer to [12] (see also [14]) for a proof. We consider the question for higher dimensions. In dimension 3 or above, a knight moves by two steps along one coordinate and by one step along a different one, we refer to Section 2 for a more rigorous definition. Stewart [13] and DeMaio [2] constructed some examples of 3-dimensional knight tours. Then, in 2011, in [3], DeMaio and Mathew extended Theorem 1 by classifying all the 3-dimensional rectangular chessboards which admit a knight tour.

Theorem 2 (DeMaio and Mathew). Let $2 \leqslant n \leqslant m \leqslant p$. The $n \times m \times p$ chessboard has a closed knight tour if and only if the following conditions hold:

1. $m, n$, or $p$ is even,

2. $m \geqslant 3$,

3. $p \geqslant 4$. 
The proof of both of these results follow the same idea, a small number of tours are constructed which have specific structural qualities which allow them to be combined to make larger tours. In this paper we extend the previous results to higher dimensional boards using a similar idea, which we will illustrate with an example using Figure 1, a more detailed discussion will follow in Section 3.

Within the tour we first identify two patterns, which we will refer to as sites, see Figure 2.

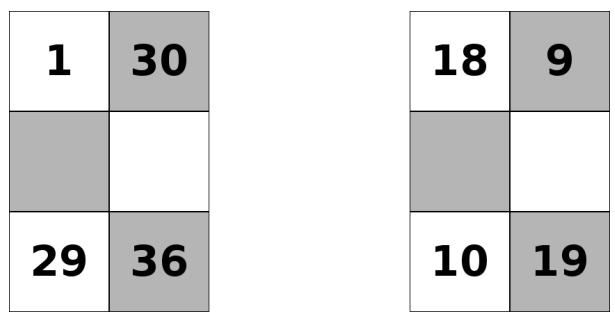

Figure 2: Two sites

If we place two copies of Figure 1 directly on top of each other, denoting the squares in the first copy $(1)_{1}$ to $(36)_{1}$ in the order they appear in the tour and similar from $(1)_{2}$ to $(36)_{2}$ in the second copy, we see that the squares $(1)_{1}$ and $(36)_{1}$ are a knight's move away from the squares $(29)_{2}$ and $(30)_{2}$ respectively. Hence we can construct a tour on a $6 \times 6 \times 2$ chessboard as follows: starting at $(1)_{1}$ we follow our original tour on the first copy until we get to $(36)_{1}$, from there we can move to $(30)_{2}$ and follow the tour on the second copy until we get to $(29)_{2}$, which is adjacent to $(1)_{1}$ and so we can close the tour. The new tour can be found in Figure 3. The first picture shows the two copies of the tour with the adjacent squares highlighted in red and green respectively. The second picture shows the new tour once the site has been glued, with the left board representing the top layer of the $6 \times 6 \times 2$ chessboard, and the right the bottom.

We refer to this process as gluing the two sites together. In order to construct a tour on a $6 \times 6 \times k$ chessboard for general $k$ however we can't just do the same thing $k$ times, because in order to join the first and second tour we've in some some way used up that site. However since we had two sites in our original tour we can simply alternate the sites we use to glue each tour together. If we denote by $\left[p_{1}, i\right]$ and $\left[p_{2}, i\right]$ the copies of the sites in the $i$ th tour we can construct a tour of a $6 \times 6 \times k$ chessboard by taking $k$ copies of Figure 1 and gluing $\left[p_{1}, 1\right]$ to $\left[p_{1}, 2\right]$, then $\left[p_{2}, 2\right]$ to $\left[p_{2}, 3\right]$ and so on until we have a tour of the entire board. Notice that this tour still contains two sites we haven't used, one in the 1st tour and one in the $k$ th, and so we could use this new tour in turn to construct a tour on $6 \times 6 \times k \times l$ chessboard in the same manner, this idea is explained in more detail in Proposition 12.

Our strategy is then as follows, we construct low dimensional tours which each contain two sites and then inductively show we can construct tours in higher dimensions by gluing these tours together. The main result in our paper is as follows:

Theorem 3. Let $2 \leqslant n_{1} \leqslant n_{2} \leqslant \ldots \leqslant n_{r}$, with $r \geqslant 3$. The $n_{1} \times \ldots \times n_{r}$ chessboard has a closed knight tour if and only if the following conditions hold: 


\section{Some $n_{i}$ is even,}

2. $n_{r-1} \geqslant 3$,

3. $n_{r} \geqslant 4$.

Note that the hypotheses are the same as the ones given in Theorem 2 when $r=3$. In the same paper they asked about higher dimensional tours, this question was also asked

\begin{tabular}{|c|c|c|c|c|c|c|c|c|}
\hline & & & 1 & 30 & 33 & 16 & 3 & 24 \\
\hline & & & 32 & 17 & 2 & 23 & 34 & 15 \\
\hline & & & 29 & 36 & 31 & 14 & 25 & 4 \\
\hline 1 & 30 & 3 & 18 & 9 & 6 & 35 & 22 & 13 \\
\hline 32 & 17 & 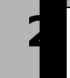 & 7 & 28 & 11 & 20 & 5 & 26 \\
\hline 29 & 36 & 3 & 10 & 19 & 8 & 27 & 12 & 21 \\
\hline 18 & 9 & & & 22 & צז & & & \\
\hline 7 & 28 & 11 & 20 & 5 & 26 & & & \\
\hline 10 & 19 & 8 & 27 & 12 & 21 & & & \\
\hline
\end{tabular}

\begin{tabular}{|c|c|c|c|c|c|}
\hline 1 & 30 & 33 & 16 & 3 & 24 \\
\hline 32 & 17 & 2 & 23 & 34 & 15 \\
\hline 29 & 36 & 31 & 14 & 25 & 4 \\
\hline 18 & 9 & 6 & 35 & 22 & 13 \\
\hline 7 & 28 & 11 & 20 & 5 & 26 \\
\hline 10 & 19 & 8 & 27 & 12 & 21 \\
\hline
\end{tabular}

\begin{tabular}{|l|l|l|l|l|l|}
\hline 44 & 37 & 40 & 59 & 46 & 67 \\
\hline 39 & 60 & 45 & 66 & 41 & 58 \\
\hline 72 & 43 & 38 & 57 & 68 & 47 \\
\hline 61 & 52 & 49 & 42 & 65 & 56 \\
\hline 50 & 71 & 54 & 63 & 48 & 69 \\
\hline 53 & 62 & 51 & 70 & 55 & 64 \\
\hline
\end{tabular}

Figure 3: New tour obtained by gluing 
by DeMaio [2] and Watkins [14]. We mention that a conjecture for this theorem was given in $[9]$.

The paper is organized as follows. In Section 2 we set the notation and define the graph structure induced by a knight on an $n$ dimensional board. Then, in Section 3 we introduce the notion of a site and explain in detail how to glue two tours together. In Section 4 we use these idea to prove Theorem 3. In Section 5 we show we can apply this technique to the problem of knight tours with more general moves and mention a conjecture of our own.

\section{Notation}

In graph theoretical terms we can consider an $n \times m$ chessboard as a grid of $n \times m$ points. We associate with this grid a graph, the knight graph $K(n, m)$, where each point is joined to all points a knight's move away. Equivalently:

Definition 4. $G=K(n, m)$ is the graph where $V(G)=\{(i, j): 1 \leqslant i \leqslant n, 1 \leqslant j \leqslant m\}$ and $((i, j),(k, l)) \in E(G) \Leftrightarrow(i-k, j-l) \in\{( \pm 1, \pm 2),( \pm 2, \pm 1)\}$.

So a closed knight tour on an $n \times m$ chessboard is precisely a Hamiltonian cycle in $K(n, m)$. We will only consider closed tours in this paper and so for brevity's sake will refer to a closed knight's tour on an $n \times m$ chessboard as simply an $n \times m$ tour.

Beyond this we can define the knight graph for higher dimensional chessboards. For a board with dimensions $n_{1} \times n_{2} \ldots \times n_{r}$, we define $K\left(n_{1}, n_{2} \ldots, n_{r}\right)$ in a similar fashion.

Definition 5. $G=K\left(n_{1}, \ldots, n_{r}\right)$ is the graph where

$$
V(G)=\left\{\left(i_{1}, \ldots, i_{r}\right): 1 \leqslant i_{j} \leqslant n_{j} \text { for all } j\right\}
$$

and

$$
\begin{aligned}
E(G)= & \left\{\left(\left(a_{1}, \ldots, a_{r}\right),\left(b_{1}, \ldots, b_{r}\right)\right): \text { there exists } i_{1}, i_{2}\right. \text { such that } \\
& \left.\left|a_{i_{1}}-b_{i_{1}}\right|=1,\left|a_{i_{2}}-b_{i_{2}}\right|=2 \text { and } a_{j}=b_{j} \text { for all } j \neq i_{1}, i_{2}\right\} .
\end{aligned}
$$

We note that $K\left(n_{1}, \ldots, n_{r}\right)$ is bi-partite, e.g., consider the map $\phi: K\left(n_{1}, \ldots, n_{r}\right) \rightarrow$ $\{-1,1\}$ given by $\phi\left(a_{1}, \ldots, a_{n_{r}}\right):=(-1)^{a_{1} \times \ldots \times a_{n_{r}}}$ which assigns the color black or white to each square. Where the context is clear we will denote vertices of $K\left(n_{1}, \ldots, n_{r}\right)$ as vectors $\underline{a} \in \mathbb{R}^{r}$.

Remark 6. Given $n_{1}, \ldots n_{r}$ and $\psi$ a permutation on $[1, r]$ it is clear that $K\left(n_{1}, \ldots, n_{r}\right)$ is isomorphic to $K\left(n_{\psi(1)}, \ldots, n_{\psi(r)}\right)$ and so $K\left(n_{1}, \ldots, n_{r}\right)$ contains a Hamiltonian cycle if and only if $K\left(n_{\psi(1)}, \ldots, n_{\psi(r)}\right)$ does. 


\section{Looking for patterns}

We will first discuss how we glue together 2 dimensional boards before giving more general definitions so as to familiarise the reader with the concepts. Given an $n \times m$ chessboard, we say that a pair of edges $\left(\left(a_{1}, a_{2}\right),\left(b_{1}, b_{2}\right)\right)$ and $\left(\left(c_{1}, c_{2}\right),\left(d_{1}, d_{2}\right)\right)$ in a tour is a site if

$$
\left(\left|a_{1}-c_{1}\right|,\left|a_{2}-c_{2}\right|\right)=\left(\left|b_{1}-d_{1}\right|,\left|b_{2}-d_{2}\right|\right) \in\{(0,2),(2,0)\}
$$

or if

$$
\left(\left|a_{1}-d_{1}\right|,\left|a_{2}-d_{2}\right|\right)=\left(\left|b_{1}-c_{1}\right|,\left|b_{2}-c_{2}\right|\right) \in\{(0,2),(2,0)\} .
$$

Roughly speaking, there is a pairing of the endpoints of the edges such that the pairs are each two squares away from each other. Up to orientation, we give examples from Figure 1 of the three possible configurations for sites in Figure 4.
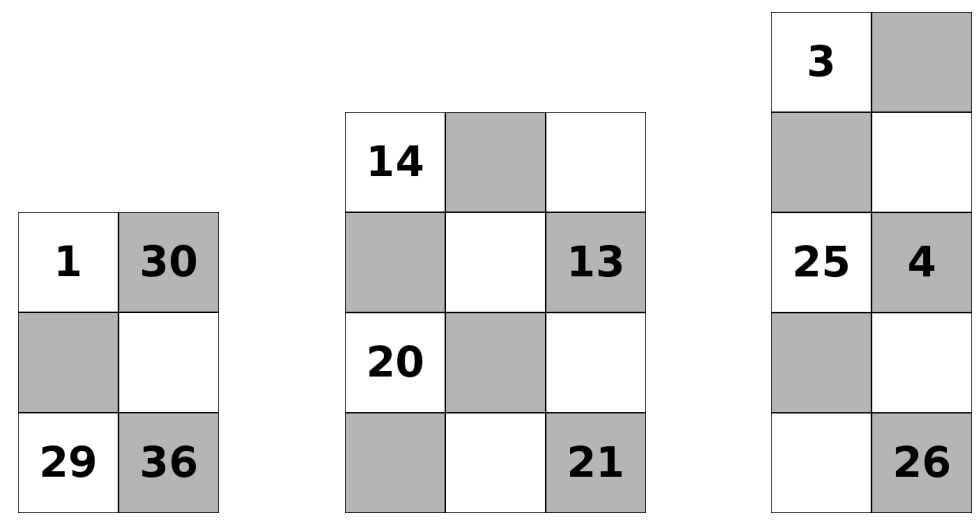

Figure 4: Sites in dimension 2.

As we saw in the introduction we can use sites to join together two knight tours and gain a dimension. If we have an $n \times m$ tour which contains a site, that is a pair of edges without loss of generality satisfying 3 . Then if we place two copies of the tour on top of each other we see that 3 guarantees that the two points $\left(a_{1}, a_{2}, 1\right)$ and $\left(c_{1}, c_{2}, 2\right)$ are adjacent in $K(n, m, 2)$, as are $\left(b_{1}, b_{2}, 1\right)$ and $\left(d_{1}, d_{2}, 2\right)$. So if we remove the edges $\left(\left(a_{1}, a_{2}, 1\right),\left(b_{1}, b_{2}, 1\right)\right)$ and $\left(\left(c_{1}, c_{2}, 2\right),\left(d_{1}, d_{2}, 2\right)\right)$ and add in the edges $\left(\left(a_{1}, a_{2}, 1\right),\left(c_{1}, c_{2}, 2\right)\right)$ and $\left(\left(b_{1}, b_{2}, 1\right),\left(d_{1}, d_{2}, 2\right)\right)$ then we have a tour on $n \times m \times 2$. The process can be thought of as in Figure 5.

We have taken two cycles and removed a single edge from each, the red edges in Figure 5 , leaving us with two paths. The definition of a site guarantees that there is a pairing of the endpoints of these paths such that the pairs are each a knight's move apart. By adding in the green edges in Figure 5, the two options corresponding to the two possible way to pair the endpoints, we form a larger cycle on the combined vertex set of the original two cycles. We refer to this process as gluing the two sites together.

We now embed the 2-dimensional case into $r$-dimensions. The basic idea will be to consider a pair of edges a site if they are a site when restricted to some 2-dimensional subspace of the $n_{1} \times \ldots \times n_{r}$ chessboard. We denote by $\left(e_{i}\right)_{i \in[1, r]}$ the canonical basis of $\mathbb{R}^{r}$. 


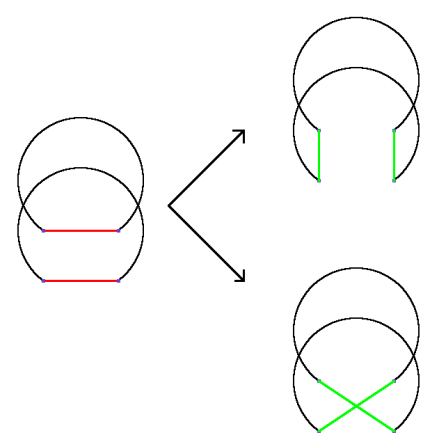

Figure 5: Gluing two sites

Definition 7. We say that a pair of edges $\{(\underline{a}, \underline{b}),(\underline{c}, \underline{d})\}$ in $K\left(n_{1}, \ldots, n_{r}\right)$ is a site if there exist $i_{1} \neq i_{2}$ such that:

$$
a_{j}=c_{j} \text { and } b_{j}=d_{j} \text {, for all } j \notin\left\{i_{1}, i_{2}\right\}
$$

and either

$$
\left(\left|a_{i_{1}}-c_{i_{1}}\right|,\left|a_{i_{2}}-c_{i_{2}}\right|\right)=\left(\left|b_{i_{1}}-d_{i_{1}}\right|,\left|b_{i_{2}}-d_{i_{2}}\right|\right) \in\{(0,2),(2,0)\}
$$

or

$$
\left(\left|a_{i_{1}}-d_{i_{1}}\right|,\left|a_{i_{2}}-d_{i_{2}}\right|\right)=\left(\left|b_{i_{1}}-c_{i_{1}}\right|,\left|b_{i_{2}}-c_{i_{2}}\right|\right) \in\{(0,2),(2,0)\}
$$

holds true.

Definition 8. An $n_{1} \times \ldots n_{r}$ tour, equivalently a Hamiltonian cycle on $K\left(n_{1}, \ldots, n_{r}\right)$, is called bi-sited if it contains two edge-disjoint sites.

We now turn to the definition of adjacent sites and explain how to "glue" them.

Definition 9. We say two sites $\{(\underline{a}, \underline{b}),(\underline{c}, \underline{d})\}$ and $\{(\underline{e}, f),(g, \underline{h})\}$ in $K\left(n_{1}, \ldots, n_{r}\right)$ are adjacent if they have the same $i_{1}$ and $i_{2}$ in Definition 7 and if there exists $j \notin\left\{i_{1}, i_{2}\right\}$ such that

$$
(\underline{a}, \underline{b}, \underline{c}, \underline{d}) \in(\underline{e}, \underline{f}, \underline{g}, \underline{h}) \pm\left(e_{j}, e_{j}, e_{j}, e_{j}\right) .
$$

We note that if we have an $n_{1} \times \ldots \times n_{r}$ tour that contains a site $[p]$ and we place two copies of the tour on top of each other, denoting by $[p, i]$ the site in the $i$-th copy, so as to cover an $n_{1} \times \ldots \times n_{r} \times 2$ chessboard then $[p, 1]$ is adjacent to $[p, 2]$. This is in essence the only property of Definition 9 that we will use.

We turn to the gluing operation which was illustrated above in Figure 5.

Definition 10. Take two adjacent sites $[p]=\{(\underline{a}, \underline{b}),(\underline{c}, \underline{d})\}$ and $[q]=\{(\underline{e}, \underline{f}),(\underline{g}, \underline{h})\}$.

1. If $[p]$ satisfies condition 3 (so does $[q]$ ), we delete the edges $(\underline{a}, \underline{b})$ and $(\underline{g}, \underline{h})$ and add the edges $(\underline{a}, \underline{g})$ and $(\underline{b}, \underline{h})$. 
2. If $[p]$ satisfies condition 4 (so does $[q]$ ), we delete the edges $(\underline{a}, \underline{b})$ and $(\underline{g}, \underline{h})$ and add the edges $(\underline{a}, \underline{h})$ and $(\underline{b}, \underline{g})$.

We say that $[p]$ and $[q]$ are glued.

Remark 11. It is a simple consequence of Definitions 7 and 9 that the new edges are all knight's moves.

We note, with reference to Figure 5, that if the sites lie in disjoint cycles then gluing the two sites together will join the cycles. We illustrate the preceding definitions with an example of a $5 \times 6$ tour, given in Figure 6 .

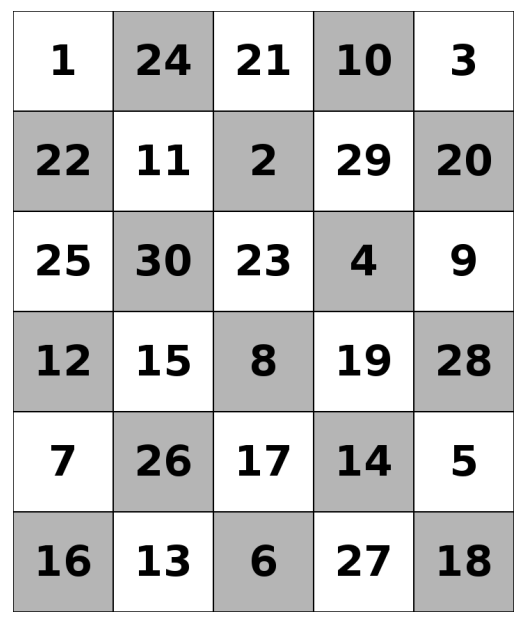

Figure 6: A $5 \times 6$ tour

In Figure 6 there is a site in the top right corner consisting of the lines $(3,4)$ and $(9,10)$. If we were to place two copies of the tour on top of each other, labelling the two tours $(1)_{i}$ to $(30)_{i}$ as in the introduction we see that the two sites $\left\{\left((3)_{1},(4)_{1}\right),\left((9)_{1},(10)_{1}\right)\right\}$ and $\left\{\left((3)_{2},(4)_{2}\right),\left((9)_{2},(10)_{2}\right)\right\}$ are adjacent. Therefore we can form a tour on the $5 \times 6 \times 2$ chessboard by gluing the two sites together, that is we remove the edges $\left((3)_{1},(4)_{1}\right)$ and $\left((9)_{2},(10)_{2}\right)$ and add in the edges $\left((3)_{1},(9)_{2}\right)$ and $\left((4)_{1},(10)_{2}\right)$.

We generalize this example to prove the following Proposition, which is the key idea in the paper.

Proposition 12. Given a bi-sited $n_{1} \times \ldots \times n_{r}$ tour and $k \geqslant 2$ then there exists a bi-sited $n_{1} \times \ldots \times n_{r} \times k$ tour .

Proof. We start by taking $k$ copies of the $n_{1} \times \ldots \times n_{r}$ tour and place them on top of each other, so as to cover the $n_{1} \times \ldots \times n_{r} \times k$ chessboard. Let us denote by $\left[p_{1}, i\right]$ and $\left[p_{2}, i\right]$ the copies of the two sites in the $i$ th copy of the tour, note that $\left[p_{j}, i\right]$ and $\left[p_{j}, i+1\right]$ are adjacent for all $1 \leqslant i \leqslant k-1,1 \leqslant j \leqslant 2$. We glue together $\left[p_{1}, 1\right]$ and $\left[p_{1}, 2\right]$ and then $\left[p_{2}, 2\right]$ and $\left[p_{2}, 3\right]$, continuing this way we glue together $\left[p_{1}, i\right]$ and $\left[p_{1}, i+1\right]$ for $i$ even and $\left[p_{2}, i\right]$ and $\left[p_{2}, i+1\right]$ for $i$ odd. We started with $k$ disjoint cycles covering $K\left(n_{1}, \ldots, n_{2}, k\right)$ 
and each time we glued two sites together we joined two cycles together. Hence at the end we are left with a Hamiltonian cycle on $K\left(n_{1}, \ldots, n_{r}, k\right)$, an $n_{1} \times \ldots \times n_{r} \times k$ tour.

It remains to check that the tour is bi-sited, and indeed there are two sites we haven't used, $\left[p_{2}, 1\right]$ and $\left[p_{2}, k\right]$ if $k$ is even and $\left[p_{2}, 1\right]$ and $\left[p_{1}, k\right]$ if $k$ is odd.

Our plan is thus to construct bi-sited tours in of small dimension and use Proposition 12 to prove Theorem 3 inductively.

\section{Into the proof}

In this section we present a proof of our main result. As mentioned our plan is first to construct bi-sited tours for low dimensional chessboards. We prove first that in 2 dimensions in fact all tours are bi-sited.

Proposition 13. Every closed tour on an $n \times m$ board is bi-sited.

Proof. First, there is no closed tour on a $2 \times m$ and on $4 \times m$ boards.

i) Case $3 \times \mathbf{m}$, for $\mathbf{m} \geqslant \mathbf{1 0}$ : Note that $m$ is even. First one has $(1,1)$ which is linked to $(3,2)$ and to $(2,3)$. The possible neighbors of $(1,3)$ are $(3,2),(2,1),(3,4),(2,5)$, see Figure 7 . As we have a cycle, exactly two of them are linked to $(1,3)$ and three of them are part of site. Then, there is at least one site. Secondly we repeat the argument for the upper right corner and get a new site. Since $m \geqslant 10$, the two sites are edge disjoint.

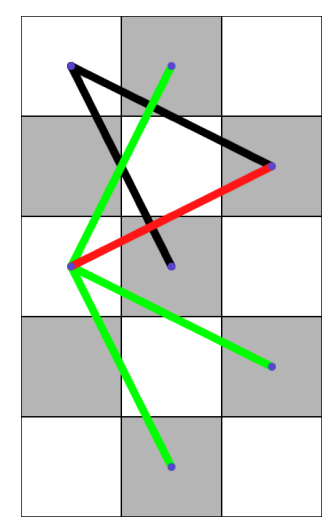

Figure 7:

ii) Case $\mathbf{n} \times \mathbf{m}$, for $\mathbf{n} \geqslant \mathbf{5}$, and $\mathbf{m} \geqslant \mathbf{6}$ : We flip the board and consider $m \times n$. We repeat twice the first part of the point i) for the sub-board of size $3 \times n$ that contains the upper left corner and for the one that contains the lower left corner. We get two disjoint sites.

Combining this with Proposition 12 already gives us a large family of high dimensional tours. 
Corollary 14. If an $n \times m$ tour exists then so does an $n \times m \times p_{1} \ldots \times p_{r}$ tour for any $p_{1}, \ldots, p_{r} \in \mathbb{N} \backslash\{0\}$.

We were not able to prove an analogue to Proposition 13 for 3 dimensional boards, however for our purposes it is enough to prove the existence of specific bi-sited tours, for which we will rely on the construction of [3].

Theorem 15. Let $2 \leqslant n \leqslant m \leqslant p$. The $n \times m \times p$ chessboard has a bi-sited tour if and only if the following conditions hold:

1. $n, m$, or $p$ is even,

2. $m \geqslant 3$,

3. $p \geqslant 4$.

Proof. It is a simple, albeit lengthy check that the tours constructed in [3] are all bi-sited. For completeness we have compiled a list of the sites with references to the Figures in [3] which can be found in Appendix A.

We are now able to prove our main result which is a clear consequence of the following theorem.

Theorem 16. Let $2 \leqslant n_{1} \leqslant n_{2} \leqslant \ldots \leqslant n_{r}$, with $r \geqslant 3$. The $n_{1} \times \ldots \times n_{r}$ chessboard has a bi-sited tour if and only if the following conditions hold:

1. Some $n_{i}$ is even,

2. $n_{r-1} \geqslant 3$,

3. $n_{r} \geqslant 4$.

We discuss the sufficient condition first.

Remark 17. We note first if condition a) does not hold then, as $K\left(n_{1}, \ldots, n_{r}\right)$ is bipartite, no tour can exist by a simple parity consideration. Now if neither $b$ ) nor $c$ ) holds true, notice that $(2,2, \ldots, 2)$ is not connected to $(2,2, \ldots, 1)$.

Proof. Keeping in mind the preceding remark, we focus on the necessary condition. We proceed by induction on $r$. The case $r=3$ is the content of Theorem 15. Given that the result folds for all $s<r$ and given $2 \leqslant n_{1} \leqslant \ldots \leqslant n_{r}$ that satisfies the conditions of the statement we see that $2 \leqslant n_{2} \leqslant \ldots \leqslant n_{r}$ is an $r-1$ tuple which satisfies the conditions of the statement and hence by the induction hypothesis a bi-sited $n_{2} \times \ldots \times n_{r}$ tour exists. By Proposition 12 a bi-sited $n_{2} \times \ldots \times n_{r} \times n_{1}$ tour exists, and by so Remark 6 a bi-sited $n_{1} \times n_{2} \times \ldots \times n_{r}$ tour exists.

An immediate consequence of Theorem 16 and of Remark 17 is:

Corollary 18. Let $2 \leqslant n_{1} \leqslant \ldots \leqslant n_{r}, r \geqslant 3$. Suppose that some $n_{i}$ is even. Then the $n_{1} \times \ldots n_{r}$ chessboard has a knight tour if and only if $K\left(n_{1}, \ldots n_{r}\right)$ is connected. 


\section{$5 \quad$ Generalised knight tours on a chessboard}

The knight's tour is a specific case of many general questions. A natural one to ask would be, what about more general moves? For example instead of the knight being able to move $( \pm 1, \pm 2)$ or $( \pm 2, \pm 1)$ what if the knight could move $( \pm \alpha, \pm \beta)$ or $( \pm \beta, \pm \alpha)$ ?

As in Section 2 we define the $(\alpha, \beta)$ knight graph on an $n_{1} \times \ldots \times n_{r}$ chessboard, the graph corresponding to all moves of a generalised knight, in the obvious way:

Definition 19. $G=K_{\alpha, \beta}\left(n_{1}, \ldots, n_{r}\right)$ is the graph where

$$
V(G)=\left\{\left(i_{1}, \ldots, i_{r}\right): 1 \leqslant i_{j} \leqslant n_{j} \text { for all } j\right\}
$$

and

$$
\begin{aligned}
E(G)= & \left\{\left(\left(a_{1}, \ldots, a_{r}\right),\left(b_{1}, \ldots, b_{r}\right)\right): \text { there exists } i_{1}, i_{2}\right. \text { such that } \\
& \left.\left|a_{i_{1}}-b_{i_{1}}\right|=\alpha,\left|a_{i_{2}}-b_{i_{2}}\right|=\beta \text { and } a_{j}=b_{j} \text { for all } j \neq i_{1}, i_{2}\right\} .
\end{aligned}
$$

We define as before an $(\alpha, \beta)$-tour on an $n_{1} \times \ldots n_{r}$ chessboard to be a Hamiltonian cycle on the graph $K_{\alpha, \beta}\left(n_{1}, \ldots, n_{r}\right)$. For brevity's sake we will refer to this as an $n_{1} \times$ $\ldots \times n_{r}(\alpha, \beta)$-tour. We refer to Figure 5 for an example.

\begin{tabular}{|c|c|c|c|c|c|c|c|c|c|}
\hline 1 & 68 & 85 & 26 & 13 & 40 & 97 & 66 & 61 & 30 \\
\hline 70 & 87 & 4 & 83 & 28 & 99 & 64 & 49 & 6 & 59 \\
\hline 25 & 12 & 41 & 2 & 67 & 62 & 31 & 14 & 39 & 96 \\
\hline 84 & 27 & 100 & 69 & 86 & 5 & 60 & 29 & 98 & 65 \\
\hline 3 & 82 & 71 & 88 & 11 & 50 & 7 & 58 & 63 & 48 \\
\hline 42 & 19 & 24 & 79 & 44 & 17 & 38 & 95 & 32 & 15 \\
\hline 89 & 74 & 53 & 22 & 81 & 36 & 93 & 76 & 51 & 34 \\
\hline 72 & 45 & 10 & 55 & 20 & 91 & 78 & 47 & 8 & 57 \\
\hline 23 & 80 & 43 & 18 & 75 & 52 & 33 & 16 & 37 & 94 \\
\hline 54 & 21 & 90 & 73 & 46 & 9 & 56 & 35 & 92 & 77 \\
\hline
\end{tabular}

Figure 8: A $10 \times 10(2,3)$-tour constructed by A. H. Frost [5] 
Remark 20. If an $n_{1} \times \ldots \times n_{r}(\alpha, \beta)$-tour exists then $\alpha$ and $\beta$ must be coprime. Indeed, $(1,1, \ldots, 1)$ is connected to $(2,1,1 \ldots, 1)$. Therefore, there are $k, l \in \mathbb{Z}$ such that $k \alpha+l \beta=$ 1 .

Our plan is to extend the concept of sites to this setting and show the methods of Section 3 can be used to obtain an analogue of Proposition 12 for $(\alpha, \beta)$-tours. As before it will be instructive to consider examples in small dimensions first. Figure 5 shows a $10 \times 10(2,3)$-tour. If we look at the edges $(1,2)$ and $(25,26)$ we see that the squares 1 and 26 and the squares 2 and 25 are both three squares away from each other. Therefore if we placed three copies of Figure 5 on top of each other, denoting the squares in the $i$ th copy $(1)_{i}$ to $(100)_{i}$ in the order they appear in the tour, we see that the squares $(1)_{1}$ and $(26)_{3}$ are a $(2,3)$ knight move away, as are the squares $(2)_{1}$ and $(25)_{3}$. Therefore we could form an $(2,3)$-tour of the first and third layers by starting at $(1)_{1}$, moving to $(26)_{3}$ and following the third tour to $(25)_{3}$, then moving to $(2)_{1}$ and following the first tour to $(1)_{1}$. Alternatively we can view this as deleting the edges $\left.\left((1)_{1},(2)_{1}\right)\right)$ and $\left.\left((25)_{3},(26)_{3}\right)\right)$ and adding in the edges $\left.\left((1)_{1},(26)_{3}\right)\right)$ and $\left.\left((2)_{1},(25)_{3}\right)\right)$. Similarly if we looked at a pair of edges like $(30,31)$ and $(96,97)$ where the squares 30 and 96 and the squares 31 and 97 are both two squares away from each other we could use the same idea to join two cycles that are 3 layers apart.

With this in mind we extend the definitions in Section 3 in the obvious way, as before we denote by $\left(e_{i}\right)_{i \in[1, r]}$ the canonical basis of $\mathbb{R}^{r}$.

Definition 21. We say that a pair of edges $(\underline{a}, \underline{b})$ and $(\underline{c}, \underline{d})$ in $K_{\alpha, \beta}\left(n_{1}, \ldots, n_{r}\right)$ is an $\alpha$-site if there exist $i_{1}, i_{2}$

$$
a_{j}=c_{j} \text { and } b_{j}=d_{j}, \text { for all } j \notin\left\{i_{1}, i_{2}\right\}
$$

and either

$$
\left(\left|a_{i_{1}}-c_{i_{1}}\right|,\left|a_{i_{2}}-c_{i_{2}}\right|\right)=\left(\left|b_{i_{1}}-d_{i_{1}}\right|,\left|b_{i_{2}}-d_{i_{2}}\right|\right) \in\{(0, \alpha),(\alpha, 0)\}
$$

or

$$
\left(\left|a_{i_{1}}-d_{i_{1}}\right|,\left|a_{i_{2}}-d_{i_{2}}\right|\right)=\left(\left|b_{i_{1}}-c_{i_{1}}\right|,\left|b_{i_{2}}-c_{i_{2}}\right|\right) \in\{(0, \alpha),(\alpha, 0)\}
$$

holds true.

Definition 22. We say two $\alpha$-sites $\{(\underline{a}, \underline{b}),(\underline{c}, \underline{d})\}$ and $\{(\underline{e}, \underline{f}),(\underline{g}, \underline{h})\}$ in $K_{\alpha, \beta}\left(n_{1}, \ldots, n_{r}\right)$ are adjacent if they have the same $i_{1}$ and $i_{2}$ in Definition 21 and if there exists $j \notin\left\{i_{1}, i_{2}\right\}$ such that

$$
(\underline{a}, \underline{b}, \underline{c}, \underline{d}) \in(\underline{e}, \underline{f}, \underline{g}, \underline{h}) \pm\left(\beta e_{j}, \beta e_{j}, \beta e_{j}, \beta e_{j}\right) .
$$

We note that if we have an $n_{1} \times \ldots \times n_{r}(\alpha, \beta)$-tour that contains an $\alpha$-site $[p]$ and we place $\beta+1$ copies of the tour on top of each other, denoting by $[p, i]$ the site in the ith copy, so as to cover an $n_{1} \times \ldots \times n_{r} \times(\beta+1)$ chessboard then $[p, 1]$ is adjacent to $[p, \beta+1]$. Again this is the only property of Definition 22 that we will use.

Definition 23. Take two adjacent $\alpha$-sites $[p]=\{(\underline{a}, \underline{b}),(\underline{c}, \underline{d})\}$ and $[q]=\{(\underline{e}, \underline{f}),(\underline{g}, \underline{h})\}$. 
1. If $[p]$ satisfies condition 5 , we delete the edges $(\underline{a}, \underline{b})$ and $(\underline{g}, \underline{h})$ and add the edges $(\underline{a}, \underline{g})$ and $(\underline{b}, \underline{h})$.

2. If $[p]$ satisfies condition 6 , we delete the edges $(\underline{a}, \underline{b})$ and $(\underline{g}, \underline{h})$ and add the edges $(\underline{a}, \underline{h})$ and $(\underline{b}, \underline{g})$.

We say that $[p]$ and $[q]$ are glued.

We note, again with reference to Figure 5 , that if the sites lie in disjoint cycles then gluing the two sites together will join the cycles. The corresponding definitions for $\beta$-sites are defined in the obvious manner.

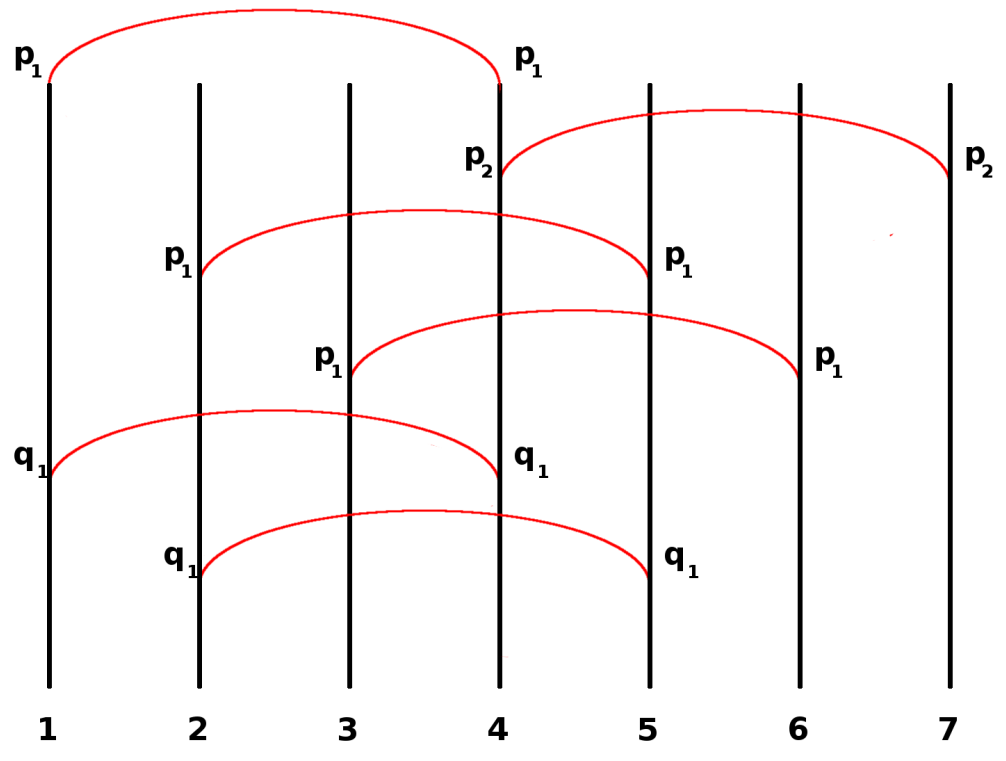

Figure 9: Construction of a $10 \times 10 \times 7(2,3)$ tour

If we want to use these ideas to construct a $10 \times 10 \times k(2,3)$-tour in a similar manner to Proposition 12 we have to be a bit cleverer. We give an example with $k=7$, note that Figure 5 contains two 2-sites and two 3-sites, all of which are edge disjoint. $p_{1}=\{(1,2),(25,25)\}, p_{1}=\{(30,31),(96,97)\}, q_{1}=\{(53,54),(89,90)\}$, and $q_{2}=\{(34,35),(76,77)\}$.

As before we can take 7 copies of Figure 5 to cover the $10 \times 10 \times 7$ chessboard, but now we can only use 2-sites and 3 -sites to join cycles which are 3 and 2 layers apart respectively. So, in the manner of Proposition 12, we can use our 2-sites to construct a cycle on the layers $\{1,4,7\}$, and similarly on the layers $\{2,5\}$ and the layers $\{3,6\}$. At this point we have three disjoint cycles which cover the $10 \times 10 \times 7$ chessboard and also, since the sites were edge disjoint, each cycle still contains both the 3 -sites on each layer. Therefore we can use our 3-sites to join layer 1 and layer 3 and since both these sites are in different cycles this will reduce the number of cycles to two. Finally we can use the second 3 -site to join layer 3 to layer 5 , completing the tour, see Figure 9. 
For general $\alpha$ and $\beta$ our strategy will be the same, use the $\alpha$ sites to construct a cycle on the layers $\{i, \beta+i, 2 \beta+i, \ldots\}$ for $1 \leqslant i \leqslant \beta$, then use the $\beta$ sites to join these cycles together. With these ideas we can prove an analogue of Proposition 12 for $(\alpha, \beta)$-tours.

Proposition 24. Given $2 \leqslant n_{1} \ldots \leqslant n_{r}$ and $k \geqslant \alpha+\beta+1$. Suppose that the $n_{1} \times \ldots \times$ $n_{r}$ chessboard has an $(\alpha, \beta)$-tour which contains $2 \alpha$-sites and $2 \beta$-sites, which are edge disjoint. Then the $n_{1} \times \ldots \times n_{r} \times k$ chessboard has an $(\alpha, \beta)$-tour which contains $2 \alpha$-sites and $2 \beta$-sites, which are edge disjoint.

Proof. Let $\alpha>\beta$, recall that by Remark $20 \alpha$ and $\beta$ are coprime. As before we start by taking $k$ copies of the $n_{1} \times \ldots \times n_{r}(\alpha, \beta)$-tour, so as to cover the $n_{1} \times \ldots \times n_{r} \times k$ chessboard. We note at this stage we have $k$ disjoint cycles covering $K_{\alpha, \beta}\left(n_{1}, \ldots, n_{r}, k\right)$. Let us denote by $\left[p_{1}, i\right]$ and $\left[p_{2}, i\right]$ the two $\alpha$-sites in the $i$ th tour and similar by $\left[q_{1}, i\right]$ and $\left[q_{2}, i\right]$ the two $\beta$-sites.

As in Proposition 12 we glue $\left[p_{1}, 1\right]$ and $\left[p_{1}, \beta+1\right]$, then $\left[p_{2}, \beta+1\right]$ and $\left[p_{2}, 2 \beta+1\right]$ and so on until we have a single cycle $\mathcal{C}_{1}$ which covers the layers

$$
\bigcup_{i \equiv 1}\left(n_{1} \times \ldots \times n_{r}, i\right)
$$

We do the same thing starting at $\left[p_{1}, i\right]$ for all $i \leqslant \beta$. At this point we have $\beta$ disjoint cycles, $\mathcal{C}_{1}, \ldots, \mathcal{C}_{\beta}$, covering $K_{\alpha, \beta}\left(n_{1}, \ldots, n_{r}, k\right)$. We note that $\mathcal{C}_{i}$ contains each $\beta$-site $\left[q_{1}, j\right]$ and $\left[q_{2}, j\right]$ such that $j \equiv i \bmod \beta$. We now use these $\beta$-sites to join $\mathcal{C}_{1}, \ldots, \mathcal{C}_{\beta}$ into a Hamiltonian cycle.

Let $d_{1}:=1$ and, for $i \in[2, \beta]$ we set $d_{i} \in[1, \beta]$ such that $d_{i}=d_{i-1}+\alpha \bmod \beta$. Since $\alpha$ and $\beta$ are coprime, we stress that $d_{i}$ is well-defined and that the map $i \mapsto d_{i}$ is a bijection onto $[1, \beta]$.

Now we glue $\left[q_{1}, 1\right]$ to $\left[q_{1}, 1+\alpha\right]$, noting that $\left[q_{1}, 1+\alpha\right]$ is in $\mathcal{C}_{d_{2}}$. We then glue $\left[q_{2}, d_{2}\right]$ to $\left[q_{2}, d_{2}+\alpha\right]$, noting that $\left[q_{2}, d_{2}\right]$ is in $\mathcal{C}_{d_{2}}$ and $\left[q_{2}, d_{2}+\alpha\right]$ is in $\mathcal{C}_{d_{3}}$. We continue as such, alternating sites, until all the cycles have been joined together. At this point we have constructed a Hamiltonian cycle on $K_{\alpha, \beta}\left(n_{1}, \ldots, n_{r}, k\right)$ and hence an $n_{1} \times \ldots \times n_{r} \times k$ $(\alpha, \beta)$-tour.

As in Proposition 12 it is a simple check that this construction leaves at least $2 \alpha$-sites and $2 \beta$-sites remaining.

We turn to the main result of this section. As in the $(1,2)$-tour case, it is sufficient to construct tours in a low dimension to show the existence of tours on all, sufficiently large, chessboards in higher dimensions.

Theorem 25. Given $\alpha>\beta, n_{1}, n_{2} \geqslant 2 \alpha+1$ and $n_{3}, \ldots, n_{k} \geqslant \alpha+\beta+1$, if the $n_{1} \times n_{2}$ chessboard has an $(\alpha, \beta)$-tour then the $n_{1} \times n_{2} \times \ldots \times n_{k}$ chessboard has an $(\alpha, \beta)$-tour.

Proof. We first note that it is a straightforward generalization of Proposition 13 that for $n_{1}, n_{2} \geqslant 2 \alpha+1$ every $(\alpha, \beta)$-tour on an $n_{1} \times n_{2}$ chessboard will contain $2 \alpha$-sites and $2 \beta$ sites. Therefore, as in the proof of Theorem 3 , the result follows directly from Proposition 24 
It is not known in general for which $\alpha, \beta(\alpha, \beta)$-tours exist on sufficiently large chessboards. Knuth showed in [8] that in 2 dimensions if $\alpha \geqslant \beta$ then $K_{\alpha, \beta}\left(n_{1}, n_{2}\right)$ is connected if and only if $\operatorname{gcd}(\alpha+\beta, \alpha-\beta)=1, n_{1} \geqslant 2 \alpha$, and $n_{2} \geqslant \alpha+\beta$. In light of the conditional nature of Theorem 25 it seems natural to conjecture

Conjecture 26. Take $\alpha, \beta$ such that $\operatorname{gcd}(\alpha+\beta, \alpha-\beta)=1$. Then, there exists $M$ such that an $(\alpha, \beta)$-tour exists on all $n_{1} \times n_{2}$ chessboards, where $n_{1}$ is even and $n_{1}, n_{2} \geqslant M$.

\section{Acknowledgements}

We would like to thank Imre Leader for useful discussions and comments on the script.

\section{A Bi-sited tours in 3 dimensions}

Without going into the details, we give a rough idea of the approach of [3]. Given an $n \times m \times p$ chessboard that satisfies the conditions a), b) and c) in Theorem 2 . Note that at least one of them is even (say $n$ ). Then an $n \times m \times p$ chessboard can be written as a union of the following ones: $2 \times 4 \times 4,2 \times 4 \times 5,2 \times 4 \times 6,2 \times 4 \times 3,2 \times 5 \times 5,2 \times 5 \times 6$, $2 \times 5 \times 3,2 \times 6 \times 6,2 \times 6 \times 3,2 \times 7 \times 3,4 \times 3 \times 3,6 \times 3 \times 3$. The list is rather long since one has no closed knight tour for $2 \times 2 \times 3$ and $2 \times 3 \times 3$ boards. The authors construct a knight tour for each elementary chessboard and describe a process for "gluing" these tours together.

We study the tours of the elementary chessboards that are exhibited in [3]. For each of them, we list two sites contained in the tour whose edges are not used in the process of joining two tours together.

\begin{tabular}{|c|c|c|}
\hline Figure & Chessboard & Sites \\
\hline Figure 6 & $2 \times 4 \times 4$ & $(4,5,11,12),(1,2,21,22)$ \\
Figure 11 & $2 \times 4 \times 5$ & $(1,2,30,31),(19,20,24,25)$ \\
Figure 14 & $2 \times 4 \times 6$ & $(7,8,26,27),(14,15,46,47)$ \\
Figure 17 & $2 \times 4 \times 3$ & $(8,9,21,22),(9,10,16,17)$ \\
Figure 20 & $2 \times 5 \times 5$ & $(8,9,25,26),(12,13,45,46)$ \\
Figure 26 & $2 \times 5 \times 3$ & $(3,4,21,22),(9,10,15,16)$ \\
Figure 29 & $2 \times 6 \times 6$ & $(16,17,57,58),(23,24,69,70)$ \\
Figure 32 & $2 \times 6 \times 3$ & $(6,7,15,16),(8,9,17,18)$ \\
Figure 35 & $2 \times 7 \times 3$ & $(2,3,25,26),(6,7,19,20)$ \\
Figure 39 & $4 \times 3 \times 3$ & $(3,4,35,36),(11,12,15,16)$ \\
Figure 40 & $6 \times 3 \times 3$ & $(2,3,5,6),(10,11,15,16)$ \\
\hline
\end{tabular}

We have not included Figure 23 of [3], the $2 \times 5 \times 6$ chessboard, since this is in fact not a cycle. Indeed, one sees that 1 cannot be reached from 60 . To fix this, we propose to take:

We replace the table following the board, i.e., the table on the top of page 9 . By 


\begin{tabular}{|c|c|c|c|c|}
\hline 1 & 44 & 51 & 58 & 39 \\
\hline 54 & 49 & 36 & 43 & 52 \\
\hline 45 & 60 & 53 & 50 & 57 \\
\hline 24 & 7 & 14 & 21 & 32 \\
\hline 17 & 12 & 29 & 6 & 15 \\
\hline 8 & 23 & 16 & 13 & 20 \\
\hline
\end{tabular}

\begin{tabular}{|c|c|c|c|c|}
\hline 46 & 3 & 38 & 41 & 56 \\
\hline 37 & 42 & 55 & 48 & 35 \\
\hline 2 & 47 & 4 & 59 & 40 \\
\hline 9 & 26 & 31 & 34 & 19 \\
\hline 30 & 5 & 18 & 11 & 28 \\
\hline 25 & 10 & 27 & 22 & 33 \\
\hline
\end{tabular}

Figure 10: A $5 \times 6 \times 2$ bi-sited tour replacing Figure 23 of [3].

\begin{tabular}{|l|l|l|}
\hline & Delete edges & Create edges \\
\hline Vertical & $11-12$ top board, $38-39$ bottom board & $11-38,12-39$ \\
\hline Horizontal & $58-59$ left board, $47-48$ right board & $58-47,59-48$ \\
\hline Front & $27-28$ front board, $10-11$ back board & $27-10,28-11$ \\
\hline
\end{tabular}

Giving us a final line of the above table:

\begin{tabular}{|c|c|c|}
\hline Figure & Chessboard & Sites \\
\hline Figure 23 & $5 \times 6 \times 2$ & $(2,3,46,47),(7,8,23,24)$ \\
\hline
\end{tabular}

In every case there are two edge disjoint sites. Then, following the proof of Theorem 2 in [3], we get that the $n \times m \times p$ chessboard has a closed knight tour which is obtained by gluing together tours coming from the elementary chessboards that we have just discussed. Therefore, the tour will be is bi-sited.

\section{References}

[1] G. Cairns. Pillow Chess. Mathematics Magazine, 75, no. 3, 173-186, 2002.

[2] J. DeMaio. Which Chessboards have a Closed Knight's Tour within the Cube? The Electron. J. Combin., 14, 2007.

[3] J. DeMaio and B. Mathew. Which chessboards have a closed knight's tour within the rectangular prism? Electron. J. Combin., 18, 2011.

[4] L. Euler. Solution d'une question curieuse qui ne paroît soumise à aucune analyse. Histoire de l'Académie Royale des Sciences et des Belles-Lettres de Berlin, vol. 15, 310-337, 1759.

[5] M. Frolow. Les Carrés Magiques. Plate VII, 1886.

[6] M. Hindry. Arithmetics. Springer, 2011. 
[7] M. R. Keen. The knight's tour, http://www.markkeen.com/knight/index.html

[8] D. E. Knuth. Leaper Graphs. Math. Gazette, 78, 274-297, 1994.

[9] A. Kumar. Magic Knight's Tours in Higher Dimensions. arXiv:1201.0458.

[10] I. Parberry. An Efficient Algorithm for the Knight's Tour Problem. Discrete Applied Mathematics, Vol. 73, 251-260, 1997.

[11] Y. Qing and J. J. Watkins. Knight's Tours for Cubes and Boxes. Congressus Numerantium, 181, 41-48, 2006.

[12] A. J. Schwenk. Which Rectangular Chessboards have a Knight's Tour? Mathematics Magazine, 64:5, 325-332, 1991.

[13] I. Stewart. Solid Knight's Tours. Journal of Recreational Mathematics, Vol. 4 (1), 1971.

[14] J. J. Watkins. Across the board: the mathematics of chessboard problems. Princeton University Press, Princeton, NJ, ISBN: 0-691-11503-6, 2004.

[15] J. J. Watkins. Knight's tours on cylinders and other surfaces. Congr. Numer., 143, 117-127, 2000.

[16] J. J. Watkins. Knight's tours on a torus. Mathematics Magazine, 70:3, 175-184, 1997. 